\title{
The association between thiazolidinediones and hospitalisation for fracture in type 2 diabetic patients: a Taiwanese population-based nested case-control study
}

\author{
F.-Y. Hsiao • C. D. Mullins
}

Received: 3 September 2009 / Accepted: 29 October 2009 / Published online: 27 November 2009

(C) Springer-Verlag 2009

\begin{abstract}
Aims/hypothesis Evidence from the USA has emerged that thiazolidinediones may have a negative effect on the skeleton and increase the risk of fracture, but the association between thiazolidinediones use and fractures has not been evaluated in an Asian population. Using the 2000-2005 Taiwan National Health Insurance claims database, this Taiwanese populationbased nested case-control study explored the association between thiazolidinediones use and hospitalisation for bone fracture in type 2 diabetic patients.

Methods In the study cohort of type 2 diabetic patients, we identified 18,003 patients with fracture and 90,015 matched controls. Multivariable conditional logistic regressions were used to estimate the association between exposure to thiazolidinediones and fractures. Duration of thiazolidinediones use was defined on the basis of cumulative days of exposure to thiazolidinediones during the year prior to the index date, i.e. $<30$ days, 30 to 180 days and $>180$ days. Results More type 2 diabetic patients with fractures than controls used thiazolidinediones (fractures $5.99 \%$ vs control $4.06 \%$ ). Thiazolidinediones use was associated with hospitalisation for fracture and the association was stronger with longer term exposure to thiazolidinediones $(<30$ days OR 1.32 [95\% CI 1.09-1.54], $p=0.005 ; 30-180$ days 1.42 [1.24-1.62], $p<0.0001$; and $>180$ days 1.54 [1.37-1.74], $p<0.0001)$. This dose-response relationship was significantly evident in women ( $<30$ days, 1.20 [0.93-1.55], $p=0.17$; 30-180 days, 1.57 [1.32-1.86], $p<0.0001$; and $>180$ days, 1.76 [1.52-2.04], $p<0.0001$ ), but not in men.
\end{abstract}

F.-Y. Hsiao $(\bowtie) \cdot$ C. D. Mullins

Pharmaceutical Health Services Research Department,

University of Maryland School of Pharmacy,

220 Arch Street, 12th Floor,

Baltimore, MD 21201, USA

e-mail: shsiao@rx.umaryland.edu
Conclusions/interpretation Long-term exposure of type 2 diabetic patients to thiazolidinediones was associated with higher odds of fractures among women without a significant increase in odds of fractures among men.

Keywords Case-control study · Fracture

Thiazolidinediones · Type 2 diabetes mellitus · TZDs

$\begin{array}{ll}\begin{array}{l}\text { Abbreviations } \\ \text { ADOPT }\end{array} & \begin{array}{l}\text { A Diabetes Outcome and Progression } \\ \text { Trial }\end{array} \\ \text { COPD } & \begin{array}{l}\text { Chronic obstructive pulmonary disease } \\ \text { ICD-9 CM }\end{array} \\ \begin{array}{l}\text { International Classification of Diseases, } \\ \text { 9th edition }\end{array} \\ \text { NHI } & \text { National Health Insurance }\end{array}$

\section{Introduction}

Type 2 diabetes patients have a higher risk of fractures; however, it is unclear whether the increased risk is attributable to patient genetic and demographic factors or treatment of the underlying diseases [1]. Recent evidence has emerged that the widely used thiazolidinediones, oral hypoglycaemic agents known as insulin sensitisers that are often prescribed when other medications have failed to reduce blood glucose levels in patients with type 2 diabetes, may have a negative effect on the skeleton and increase the risk of fracture [2]. Since 2004, preclinical [3-7] and clinical studies [8-10] have indicated that thiazolidinediones use could alter bone metabolism, resulting in reduced osteoblastic bone formation and accelerated bone loss. As suggested by recent evidence from an epidemiological study [9] and a clinical trial [11], thiazolidinediones 
use is associated with a decrease in bone density in postmenopausal women. Similar findings have been reported in older men with type 2 diabetes [8]. However, most studies to date have addressed the impact of thiazolidinediones use on bone turnover or bone loss in clinical trials. Evidence on how their results link to fracture risk is somewhat limited.

Evidence that thiazolidinediones increase fracture risk emerged with the adverse events reports of the A Diabetes Outcome and Progression Trial (ADOPT) [11, 12] published in 2006. Although a case-control study by Meier et al. [13] provided further evidence of a possible association between thiazolidinediones use and fractures in patients with diabetes mellitus, a dose-response relationship was not confirmed due to the relatively few patients using thiazolidinediones in case and control groups. In addition, the question of whether there is a sex-related difference in the skeletal response to thiazolidinediones was left unanswered. So far, the negative skeletal effects seem to be more significant for women than for men, but results are not conclusive. Findings from ADOPT [12] indicated that the increased risk of fractures occurred only in women. In contrast, an analysis [13] using data derived from the UKbased General Practice Research Database (GPRD) showed that the fracture risk also increased in men. Racial/ethnic differences in fracture risk have been shown in the general population [14], but no study has evaluated the association between fracture risk and thiazolidinediones use in the Asian population.

Additional studies are warranted as evidence surrounding the fracture risk among thiazolidinediones users stratified by race/ethnicity and sex is limited and studies examining the correlation between length of exposure to thiazolidinediones and fractures are lacking. Specifically, a dose-response relationship between fracture risk and thiazolidinediones use would provide additional evidence and strengthen the proposed relationship. Therefore, using Taiwan's National Health Insurance (NHI) claims database, this large population-based nested case-control study was conducted to explore the dose-response relationship between thiazolidinediones use and risk of fracture in type 2 diabetic patients.

\section{Methods}

Data source Taiwan's mandatory NHI programme, with approximately 23 million insured persons, covers over $99 \%$ of Taiwan's population. The Taiwanese NHI offers complete freedom of choice among healthcare providers contracted with the NHI and comprehensive benefits including inpatient care, ambulatory care, dental care and prescription drugs to beneficiaries. The NHI database contains administrative claims that reflect resource use, including physician visits, hospital care and prescribed medications. The completeness and accuracy of the NHI database are ensured by the Department of Health and the Bureau of NHI of Taiwan [15]. The longitudinal nature of NHI's database permits identification of cohorts based upon diagnoses, health services accessed, drugs used, medical history, prescription drug profile and endpoint of drug treatment.

Study population We identified in the Taiwanese NHI database all individuals who had their first ambulatory visit with a diagnosis of diabetes (International Classification of Diseases, 9th edition [ICD-9 CM; www.icd9data.com/2007/ Volume1/240-279/250-259/250/default.htm], code: 250.xx) and received at least one prescription for an oral hypoglycaemic agent (thiazolidinediones [rosiglitazone and pioglitazone], biguanide metformin, sulfonylurea derivative, prandial glucose regulator [repaglinide] or alphaglucosidase inhibitor acarbose) between 2001 and 2005 $(n=511,533)$. Patients were excluded if they had type 1 diabetes (ICD-9 CM codes: 250.x1). Because patients receiving insulin tend to have either more advanced type 2 diabetes mellitus or type 1 diabetes mellitus, patients receiving insulin at cohort entry were excluded. However, those who received insulin after the initial oral hypoglycaemic agent treatment during the follow-up were retained in our study. The cohort entry date for each patient was defined as the date that an oral hypoglycaemic agent was first prescribed.

Case patient definition and control participants selection In this study population of type 2 diabetic patients, we identified all patients hospitalised with a primary diagnosis of fracture (ICD-9 CM code) between 2001 and 2005. Only the first hospitalisation for fracture was included in our study. Matched controls were chosen at random from the same type 2 diabetic patient cohort. We identified five control participants per case patient. Controls were matched to case patients for age (same year of birth) and sex as well as past medical history, including prior fracture, Paget's disease, osteoporosis, rheumatoid arthritis or osteoarthritis in the prior year, and use of bisphosphates, hormone replacement therapy or corticosteroid in the prior year. We defined an index date for all participants as the date of hospitalisation for fracture for case patients or a random date within the range of the case index dates for controls.

Exposure to thiazolidinediones Using NHI database, we assessed whether patients received thiazolidinediones during the year prior to the index date. Thiazolidinediones exposure was inferred from computer-based prescription claims. Duration of thiazolidinediones use was defined according to the number of days thiazolidinediones was 
taken during the year prior to the index date. In addition, thiazolidinediones users were classified into cohorts according to their cumulative exposure, i.e. $<30$ days, 30 to 180 days and $>180$ days.

Statistical analysis Multivariable conditional logistic regression was used to estimate the association between exposure to thiazolidinediones and fractures. Pre-planned secondary analyses were conducted in subgroups stratified by sex or fracture sites. The associations are presented as ORs with 95\% CIs. The $p$ values are two-sided, with $p<$ 0.05 considered statistically significant. All models were adjusted for a severity of diabetes proxy (number of hospitalisations for diabetes in the year prior to cohort entry) and co-morbidities potentially associated with an altered fracture risk such as stroke, transient ischaemic attack, asthma or chronic obstructive pulmonary disease (COPD), or drug treatments potentially associated with an altered fracture risk (anticonvulsants, antipsychotic agents, hypnotics/sedatives, diuretics, proton-pump inhibitors and calcium/vitamin D supplements) over 1 year prior to cohort entry. The main analysis included patients with prior fractures and adjusted for these prior fractures, while a sensitivity analysis repeated the analysis excluding patients with prior fractures. All analyses were conducted using SAS software (SAS 9.2; SAS Institute, Cary, NC, USA).
Table 1 Baseline characteristics of fracture cases and their matched controls

\footnotetext{
${ }^{\mathrm{a}}$ Values mean (SD); ${ }^{\mathrm{b}}$ hospitalisation for diabetes

NA, not available
}

\begin{tabular}{|c|c|c|c|c|c|}
\hline \multirow[t]{2}{*}{ Characteristics } & \multicolumn{2}{|l|}{ Cases } & \multicolumn{2}{|c|}{ Controls } & \multirow[t]{2}{*}{$p$ value } \\
\hline & $n$ & $\%$ & $n$ & $\%$ & \\
\hline$n$ & 18,003 & & 90,015 & & \\
\hline \multicolumn{6}{|l|}{ Demographic } \\
\hline Age (years) ${ }^{\mathrm{a}}$ & 62.42 & 13.52 & 62.42 & 13.52 & NA \\
\hline Women & 9,819 & 54.54 & 49,095 & 54.54 & NA \\
\hline \multicolumn{6}{|l|}{ Diabetes severity } \\
\hline Hospitalisation in preceding year ${ }^{\mathrm{a}, \mathrm{b}}$ & 0.04 & 0.21 & 0.04 & 0.20 & 0.417 \\
\hline \multicolumn{6}{|l|}{ History of fracture in preceding year } \\
\hline All fractures & 62 & 0.34 & 310 & 0.34 & NA \\
\hline Vertebral fracture & 4 & 0.02 & 39 & 0.04 & 0.195 \\
\hline Humerus fracture & 8 & 0.04 & 0 & 0.00 & $<0.0001$ \\
\hline Wrist fracture & 6 & 0.03 & 54 & 0.06 & 0.166 \\
\hline Hip fracture & 17 & 0.09 & 128 & 0.14 & 0.110 \\
\hline Femur fracture & 1 & 0.01 & 25 & 0.03 & 0.079 \\
\hline Others & 26 & 0.14 & 64 & 0.07 & 0.002 \\
\hline \multicolumn{6}{|c|}{ Other medical conditions in preceding year } \\
\hline Paget's disease & 3 & 0.02 & 19 & 0.02 & NA \\
\hline Osteoporosis & 1,581 & 8.78 & 7,611 & 8.46 & NA \\
\hline Rheumatoid arthritis & 280 & 1.56 & 1,400 & 1.56 & NA \\
\hline Osteoarthritis & 4,036 & 22.42 & 20,180 & 22.42 & NA \\
\hline Stroke & 1,166 & 6.48 & 5,346 & 5.94 & 0.006 \\
\hline Transient ischaemic attack & 318 & 1.77 & 1,554 & 1.73 & 0.707 \\
\hline Asthma & 1,347 & 7.48 & 6,607 & 7.34 & 0.505 \\
\hline COPD & 836 & 4.64 & 3,664 & 4.07 & 0.000 \\
\hline \multicolumn{6}{|l|}{ Other treatments in preceding year } \\
\hline Bisphosphates & 46 & 0.26 & 230 & 0.26 & NA \\
\hline Hormone replacement therapy & 298 & 1.66 & 1,490 & 1.66 & NA \\
\hline Corticosteroids & 6,110 & 33.94 & 30,550 & 33.94 & NA \\
\hline Anticonvulsants & 1,573 & 8.74 & 7,445 & 8.27 & 0.039 \\
\hline Antipsychotic agents & 3,089 & 17.16 & 14,218 & 15.80 & $<0.0001$ \\
\hline Hypnotics/sedatives & 9,899 & 54.99 & 47,607 & 52.89 & $<0.0001$ \\
\hline Diuretics & 4,842 & 26.90 & 23,604 & 26.22 & 0.061 \\
\hline Proton-pump inhibitors & 783 & 4.35 & 3,706 & 4.12 & 0.154 \\
\hline Calcium/vitamin D supplement & 27 & 0.15 & 121 & 0.13 & 0.606 \\
\hline
\end{tabular}




\section{Results}

We identified 18,003 case patients with fracture and 90,015 matched controls. Overall, the age and sex distribution, and prevalence of relevant co-morbidities in case patients and controls were well matched. Compared with controls, case patients had a slightly higher prevalence of stroke and COPD, and higher use of anticonvulsants, antipsychotic agents and hypnotics/sedatives before cohort entry (Table 1).

More case patients than controls used thiazolidinediones (case 5.99\% vs control $4.06 \%$ ) at any time in the year prior to index date. Thiazolidinediones users differed from nonusers in some aspects of the case and control cohort. As expected, more thiazolidinediones users had been hospital-

Table 2 Characteristics of thiazolidinediones users vs non-users in fracture cases and matched controls

\begin{tabular}{|c|c|c|c|c|c|c|c|c|c|c|}
\hline \multirow[t]{3}{*}{ Characteristic } & \multicolumn{5}{|c|}{ Cases $(n=18,003)$} & \multicolumn{5}{|c|}{ Control $(n=90,015)$} \\
\hline & \multicolumn{2}{|l|}{ TZD user } & \multicolumn{2}{|c|}{ Non-TZD user } & \multirow[t]{2}{*}{$p$ value } & \multicolumn{2}{|l|}{ TZD user } & \multicolumn{2}{|c|}{ Non-TZD user } & \multirow[t]{2}{*}{$p$ value } \\
\hline & $n$ & $\%$ & $n$ & $\%$ & & $n$ & $\%$ & $n$ & $\%$ & \\
\hline$n, \%$ & 1,078 & 5.99 & 16,925 & 94.01 & & 3,651 & 4.06 & 86,364 & 95.94 & \\
\hline Age (years) ${ }^{\mathrm{a}}$ & 60.64 & 12.86 & 62.54 & 13.55 & 0.622 & 60.86 & 12.18 & 62.49 & 13.57 & 0.678 \\
\hline Women & 653 & 60.58 & 9,166 & 54.16 & $<0.0001$ & 2,105 & 57.66 & 46,990 & 54.41 & 0.000 \\
\hline \multicolumn{11}{|l|}{ Diabetes severity } \\
\hline $\begin{array}{l}\text { Hospitalisation in preceding } \\
\text { year }^{\mathrm{a}, \mathrm{b}}\end{array}$ & 61 & 5.66 & 636 & 3.76 & 0.002 & 151 & 4.14 & 2,782 & 3.22 & 0.002 \\
\hline \multicolumn{11}{|l|}{$\begin{array}{l}\text { History of fracture in preceding } \\
\text { year }\end{array}$} \\
\hline All fractures & 0 & 0.00 & 62 & 0.37 & 0.047 & 3 & 0.08 & 307 & 0.36 & 0.006 \\
\hline Vertebral fracture & 0 & 0.00 & 4 & 0.02 & 0.614 & 0 & 0.00 & 39 & 0.05 & 0.199 \\
\hline Humerus fracture & 0 & 0.00 & 8 & 0.05 & 0.475 & 0 & 0.00 & 0 & 0.00 & NA \\
\hline Wrist fracture & 0 & 0.00 & 6 & 0.03 & 0.536 & 0 & 0.00 & 54 & 0.06 & 0.131 \\
\hline Hip fracture & 0 & 0.00 & 17 & 0.10 & 0.298 & 2 & 0.05 & 126 & 0.15 & 0.152 \\
\hline Femur fracture & 0 & 0.00 & 1 & 0.01 & 0.801 & 0 & 0.00 & 25 & 0.03 & 0.304 \\
\hline Others & 0 & 0.00 & 26 & 0.15 & 0.198 & 1 & 0.03 & 63 & 0.07 & 0.312 \\
\hline \multicolumn{11}{|l|}{$\begin{array}{l}\text { Other medical conditions in } \\
\text { preceding year }\end{array}$} \\
\hline Paget's disease & 0 & 0.00 & 3 & 0.02 & 0.323 & 0 & 0.00 & 19 & 0.02 & 0.370 \\
\hline Osteoporosis & 73 & 6.77 & 1,508 & 8.91 & 0.016 & 232 & 6.35 & 7379 & 8.54 & $<0.0001$ \\
\hline Rheumatoid arthritis & 6 & 0.56 & 274 & 1.62 & 0.006 & 27 & 0.74 & 1,373 & 1.59 & $<0.0001$ \\
\hline Osteoarthritis & 196 & 18.18 & 3,840 & 22.69 & 0.001 & 625 & 17.12 & 19,555 & 22.64 & $<0.0001$ \\
\hline Stroke & 71 & 6.59 & 1,095 & 6.47 & 0.880 & 316 & 8.66 & 5,030 & 5.82 & $<0.0001$ \\
\hline Transient ischaemic attack & 21 & 1.95 & 297 & 1.75 & 0.641 & 74 & 2.03 & 1,480 & 1.71 & 0.155 \\
\hline Asthma & 61 & 5.66 & 1,286 & 7.60 & 0.019 & 360 & 9.86 & 6,247 & 7.23 & $<0.0001$ \\
\hline COPD & 35 & 3.25 & 801 & 4.73 & 0.025 & 91 & 2.49 & 3,573 & 4.14 & $<0.0001$ \\
\hline \multicolumn{11}{|l|}{$\begin{array}{l}\text { Other treatments in preceding } \\
\text { year }\end{array}$} \\
\hline Bisphosphates & 1 & 0.09 & 45 & 0.27 & 0.275 & 4 & 0.11 & 226 & 0.26 & 0.075 \\
\hline Hormone replacement therapy & 25 & 2.32 & 273 & 1.61 & 0.078 & 81 & 2.22 & 1,409 & 1.63 & 0.007 \\
\hline Corticosteroids & 335 & 31.08 & 5,775 & 34.12 & 0.041 & 1,150 & 31.50 & 29,400 & 34.04 & 0.002 \\
\hline Anticonvulsants & 110 & 10.20 & 1,463 & 8.64 & 0.079 & 339 & 9.29 & 7,106 & 8.23 & 0.023 \\
\hline Antipsychotic agents & 202 & 18.74 & 2,887 & 17.06 & 0.156 & 659 & 18.05 & 13,559 & 15.70 & 0.000 \\
\hline Hypnotics/sedatives & 572 & 53.06 & 9,327 & 55.11 & 0.190 & 2,064 & 56.53 & 45,543 & 52.73 & $<0.0001$ \\
\hline Diuretics & 276 & 25.60 & 4,566 & 26.98 & 0.324 & 825 & 22.60 & 22,779 & 26.38 & $<0.0001$ \\
\hline Proton-pump inhibitors & 41 & 3.80 & 742 & 4.38 & 0.365 & 201 & 5.51 & 3,505 & 4.06 & $<0.0001$ \\
\hline $\begin{array}{l}\text { Calcium/vitamin D } \\
\text { supplement }\end{array}$ & 1 & 0.09 & 26 & 0.15 & 0.617 & 8 & 0.22 & 113 & 0.13 & 0.154 \\
\hline
\end{tabular}

${ }^{\mathrm{a}}$ Values mean(SD); ${ }^{\mathrm{b}}$ hospitalisation for diabetes

TZD, thiazolidinediones 
ised for diabetes prior to cohort entry as compared with non-users in the case patients group (5.66\% vs 3.76\%; $p=$ $0.002)$ and in the controls $(4.14 \%$ vs $3.22 \% ; p=0.002)$. Thiazolidinediones users were more likely to have a history of stroke and have used hormone replacement therapy, anticonvulsants and antipsychotic agents, and hypnotics/ sedatives than non-users in the control cohort (Table 2). More than half of the thiazolidinediones users in the case patients group (56.12\%) and control group (58.31\%) were current users with mean cumulative days of exposure of 211.00 (SD 126.27) days and 196.15 (SD 113.38) days, respectively (Table 3 ).

The use of thiazolidinediones was associated with hospitalisation for fracture, after adjustments for severity of diabetes, co-morbidities and drug use potentially associated with fracture risk. Significantly, the association was stronger with longer term exposure to thiazolidinediones. The ORs at exposures of $<30$ days, 30 to 180 days and $>180$ days, were: 1.32 (95\% CI 1.09-1.54), $p=0.005$; 1.42 (1.24-1.62), $p<$ 0.0001 ; and 1.54 (1.37-1.74), $p<0.0001$, respectively. Also, the association between thiazolidinediones use and hospitalisation for fracture differed by sex, with ORs significantly evident in women $(1.20$ [95\% CI 0.93-1.55], $p=0.17$ at $<30$ days, 1.57 [1.32-1.86], $p<0.0001$ at $30-180$ days, 1.76 [1.52-2.04], $p<0.0001$ at $>180$ days) but not in men (Table 4). Adjusted ORs stratified by fracture site (Table 5) provide additional evidence that thiazolidinediones use is significantly associated with various kinds of fractures (OR 1.47 [1.34-1.62] $p<0.0001)$, the strongest association being for vertebral fractures (OR $1.70[1.31-2.20] p<0.0001)$.

\section{Discussion}

In this population-based case-control study, we provide further evidence of an increased association between fractures and use of thiazolidinediones in type 2 diabetic patients. Long-term exposure to thiazolidinediones was associated with higher odds of fracture, with the highest odds seen in thiazolidinediones users with $>180$ days of use. The dose-response association with exposure was significantly evident in women but not in men.

Our findings are consistent with research done by Meier et al. [13], which reported increasing ORs of developing a fracture in association with longer thiazolidinediones use. They reported ORs for one to seven, $8-14$ and $\geq 15$ thiazolidinediones prescriptions of 0.90 (95\% CI $0.46-$ 1.74, $p=0.75$ ), 1.85 (95\% CI $0.86-3.98, p=0.12)$ and 2.86 (95\% CI 1.57-5.22, $p<0.001)$. Our study uses a more thorough and comprehensive methodological approach by calculating exposure in terms of duration (days) of exposure, an aspect of thiazolidinediones use and fracture risk not discussed in previous studies $[12,14,16]$. Furthermore, by exploring the Taiwanese nationwide data, we had access to a larger diabetes patient cohort than found in existing studies. The large number of fractures cases may provide a more precise estimate with power to look at gradients of relative odds for different durations of exposure to thiazolidinediones. This rich data set enabled us to provide evidence of thiazolidinediones use and odds of fracture that is more relevant to real-world situations.

The use of the Taiwanese NHI dataset provides the first major report of odds of fracture among an Asian population of thiazolidinediones users. Given the common use of thiazolidinediones and relatively lower bone mass in Asian groups [14], it is important to examine the fracture risk in the potentially vulnerable Asian diabetic population. Our findings not only serve as a comparison to the Western diabetic population, but also suggest clinically relevant warnings surrounding the risk of fracture among Asian thiazolidinediones users. In addition, our study has added evidence on the sex-related difference in skeletal response to thiazolidinediones, with higher odds of fracture in women but not in men. Although a higher odds of fracture risk was found in men receiving $\geq 180$ days of thiazolidinediones, no dose-response relationship was found as in women. Also, the $\mathrm{CI}$ in men is very close to $1(1.02-1.50)$ and is therefore less clinically relevant. A difference in fracture risk by sex has also been reported in the ADOPT trial [12] and in a very recent meta-analysis done by Loke
Table 3 Thiazolidinediones exposure among fracture cases and their matched controls

${ }^{\mathrm{a}}$ Values are mean (SD)

\begin{tabular}{|c|c|c|c|c|c|}
\hline \multirow[t]{2}{*}{ Patterns of use } & \multicolumn{2}{|l|}{ Cases } & \multicolumn{2}{|l|}{ Controls } & \multirow[t]{2}{*}{$p$ value } \\
\hline & $n$ & $\%$ & $n$ & $\%$ & \\
\hline$n$ & 18,003 & & 90,015 & & \\
\hline All thiazolidinediones users & 1,078 & 100.00 & 3,651 & 100.00 & \\
\hline Current users & 605 & 56.12 & 2,129 & 58.31 & 0.775 \\
\hline Total days of prescription (days) ${ }^{\mathrm{a}}$ & 211.00 & 126.27 & 196.15 & 113.38 & 0.269 \\
\hline Past users & 473 & 43.88 & 1,522 & 41.69 & 0.775 \\
\hline Total days of prescription (days) ${ }^{\mathrm{a}}$ & 98.95 & 89.23 & 89.57 & 79.42 & 0.018 \\
\hline Time since last prescription (days) ${ }^{\mathrm{a}}$ & 161.91 & 94.47 & 164.49 & 93.07 & 0.742 \\
\hline
\end{tabular}


Table 4 Crude and adjusted odds for fracture among all thiazolidinediones users, and among men and women
${ }^{\text {a }}$ All models adjusted by diabetes severity, current TZD use and co-morbidities or drug treatments potentially associated with an altered fracture risk TZD, thiazolidinediones

\begin{tabular}{|c|c|c|c|c|c|}
\hline \multirow[t]{2}{*}{ Exposure } & \multicolumn{2}{|c|}{ Study participants } & \multicolumn{2}{|l|}{ OR $(95 \% \mathrm{CI})$} & \multirow[t]{2}{*}{$p$ value } \\
\hline & Case & Control & Crude & Adjusted $^{\mathrm{a}}$ & \\
\hline \multicolumn{6}{|l|}{ All } \\
\hline$n$ & 18,003 & 90,015 & & & \\
\hline No TZD use & 16,925 & 86,364 & & & \\
\hline \multicolumn{6}{|c|}{ Cumulative TZD use } \\
\hline$<30$ days & 209 & 738 & $1.46(1.25-1.71)$ & $1.32(1.09-1.54)$ & 0.005 \\
\hline $30-180$ days & 452 & 1,539 & $1.53(1.37-1.70)$ & $1.42(1.24-1.62)$ & $<0.0001$ \\
\hline$>180$ days & 417 & 1,374 & $1.59(1.42-1.78)$ & $1.54(1.37-1.74)$ & $<0.0001$ \\
\hline \multicolumn{6}{|l|}{ Men } \\
\hline$n$ & 8,184 & 40,920 & & & \\
\hline No TZD use & 7,759 & 39,374 & & & \\
\hline \multicolumn{6}{|c|}{ Cumulative TZD use } \\
\hline$<30$ days & 102 & 307 & $1.71(1.36-2.15)$ & $1.43(1.07-1.91)$ & 0.02 \\
\hline $30-180$ days & 173 & 648 & $1.38(1.16-1.64)$ & $1.21(0.97-1.51)$ & 0.09 \\
\hline$>180$ days & 150 & 591 & $1.31(1.09-1.58)$ & $1.23(1.02-1.50)$ & 0.03 \\
\hline \multicolumn{6}{|l|}{ Women } \\
\hline$n$ & 9,819 & 49,095 & & & \\
\hline No TZD use & 9,166 & 46,990 & & & \\
\hline \multicolumn{6}{|c|}{ Cumulative TZD use } \\
\hline$<30$ days & 107 & 431 & $1.29(1.04-1.60)$ & $1.20(0.93-1.55)$ & 0.17 \\
\hline 30-180 days & 279 & 891 & $1.64(1.43-1.88)$ & $1.57(1.32-1.86)$ & $<0.0001$ \\
\hline$>180$ days & 267 & 783 & $1.80(1.56-2.08)$ & $1.76(1.52-2.04)$ & $<0.0001$ \\
\hline
\end{tabular}

et al. [17]. The meta-analysis [17] included ten randomised controlled trials and showed a significantly increased risk of fractures among women (OR 2.23, 95\% CI 1.65-3.01, $p<$ 0.001 ) but not among men (OR 1.00, 95\% CI 0.73-1.39). Our findings, therefore, add evidence to support recent warnings [18] issued by the US Food and Drug Administration (FDA) and letters from the manufacturers [19, 20] informing healthcare providers that thiazolidinediones are linked to an increased rate of fractures in women.
Most of the recently published data have indicated that thiazolidinediones-associated fractures are restricted to the upper limb (forearm, hand and wrist) or distal lower limb (foot, ankle, fibula and tibia) [11]. Our study investigated the association between thiazolidinediones use and different fracture sites. Overall, thiazolidinediones use was significantly associated with various kinds of fractures (1.47 [95\% CI 1.34-1.62] $p<0.0001)$, including fractures clinically related to osteoporosis, such as hip or femur fractures $(1.53$
Table 5 Crude and adjusted fracture odds for thiazolidinediones use and fracture type

\begin{tabular}{|c|c|c|c|c|c|}
\hline \multirow[t]{2}{*}{ Exposure } & \multicolumn{2}{|c|}{ Study participants } & \multicolumn{2}{|l|}{ OR $(95 \% \mathrm{CI})$} & \multirow[t]{2}{*}{$p$ value } \\
\hline & Cases $(n)$ & Controls $(n)$ & Crude & Adjusted $^{\mathrm{a}}$ & \\
\hline \multicolumn{6}{|c|}{ All fractures ${ }^{\mathrm{b}}$} \\
\hline TZD use & 1,078 & 16,925 & $1.54(1.43-1.65)$ & $1.47(1.34-1.62)$ & $<0.0001$ \\
\hline \multicolumn{6}{|c|}{ Vertebral fracture ${ }^{\mathrm{b}}$} \\
\hline TZD use & 119 & 1,571 & $1.76(1.45-2.14)$ & $1.70(1.31-2.20)$ & $<0.0001$ \\
\hline \multicolumn{6}{|c|}{ Humerus fracture ${ }^{b}$} \\
\hline TZD use & 104 & 1,246 & $1.83(1.48-2.26)$ & $1.34(0.99-1.83)$ & 0.06 \\
\hline \multicolumn{6}{|c|}{ Wrist fracture $^{\mathrm{b}}$} \\
\hline TZD use & 137 & 2,496 & $1.14(0.95-1.37)$ & $1.29(1.03-1.61)$ & 0.03 \\
\hline \multicolumn{6}{|c|}{ Hip/femur fracture ${ }^{b}$} \\
\hline TZD use & 326 & 4,738 & $1.77(1.57-2.00)$ & $1.53(1.30-1.80)$ & $<0.01$ \\
\hline
\end{tabular}


$[1.30-1.80] p<0.01)$. Similar findings were found in the analysis [13] done by Meier et al. Taken together, these findings could add insight into future studies on the possible mechanism and treatments of thiazolidinedionesassociated fractures.

Some limitations of the present study should be recognised. First, one of the main inherent limitations of using claim data is the measurement error caused by using the amount of medicine prescribed to define 'exposure', which presumes patients always take the medicines that they have a prescription for. Second, in addition to duration of thiazolidinediones use, dose could be another variable that affects fracture risk. However, over $90 \%$ of our thiazolidinediones users received the standard regimen of rosiglitazone $4 \mathrm{mg}$ or pioglitazone $30 \mathrm{mg}$ per day, so there was little variation in dose among the Taiwanese population. Third, the diagnosis of bone fractures was obtained through code diagnoses at hospitalisation. As a result, some events receiving no hospitalisation were excluded. Fourth, the study does not look at long-term thiazolidinediones exposure, which is an area for future research. Fifth, although we adjusted for a wide range of potential fracture risk factors, we were not able to include variables not routinely captured in a claim database, such as certain lifestyle factors (diet or physical activity). Sixth, we did not have a clinical measure of diabetes severity such as $\mathrm{HbA}_{1 \mathrm{c}}$, which would be an improvement over the use of the prior diabetic hospitalisation variable used by us. Seventh, we included in our study patients who had a previous history of fracture, rather than excluding them as in an existing study [13]. Although we matched these case patients to controls with a history of fracture, some unobserved fracture risk that could lead to distortion of the fracture risk should be taken into account. We therefore conducted a sensitivity analysis, in which we excluded patients with history of fracture, but results remained unchanged. We thus decided to include patients with previous history of fracture in our study to increase the generalisability of our findings. The final limitation is that, due to the relatively few patients receiving pioglitazone, we were unable to compare fracture odds between rosiglitazone and pioglitazone. Given that current evidence suggests that fracture risk could be a class effect of thiazolidinediones [21], further studies are needed to examine the fracture risk in rosiglitazone and pioglitazone.

Despite these limitations, our study of a nationwide Asian diabetic population offers additional insight into the use of thiazolidinediones and odds of fractures, especially the sex-specific skeletal effect of thiazolidinediones. Longterm thiazolidinediones use increases the odds of fractures among women without a significant increase in odds of fractures among men in type 2 diabetic patients.
Conclusion This study is the first to report an association between thiazolidinediones exposure and fracture in an Asian population. Our findings extend the evidence from current literature to a real-world setting and support data from clinical trials suggesting that long-term thiazolidinediones use increases the odds of fractures among women in type 2 diabetic patients. The large sample size also allowed us to confirm an increased odds of fracture across an expanded group of fracture types, extending beyond limbs to include hip and other fractures among thiazolidinediones users.

Acknowledgements F.-Y. Hsiao is supported by Taiwan's National Science Council Grant NSC-096-2917-I-564-106. We also thank Taiwan's Department of Health (DOH) for providing a research grant (DOH97-TD-D-113-97008). C. D. Mullins received grant support from Takeda for a separate fellowship project in 2007. We also thank the Bureau of National Health Insurance (BNHI) and National Health Research Institutes (NHRI) for making their databases available for this study. We would like to thank W.-F. Huang (National Yang-Ming University, Taiwan) and Y.-W. Tsai (National Health Research Institutes, Taiwan) for providing consultation and assistance with the manuscript. No financial compensation has been given for their assistance.

Duality of interest C. D. Mullins receives grant support and consulting incoming from Bayer, Lilly, Novartis, Pfizer and sanofiaventis. C. D. Mullins also receives grant support for another postdoctoral fellowship programme from Takeda Pharmaceuticals. No funding was received for the design, analysis or write-up of this study. For F.-Y. Hsiao there is no duality of interest associated with this manuscript.

\section{References}

1. Watts NB, D'Alessio DA (2006) Type 2 diabetes, thiazolidinediones: bad to the bone? J Clin Endocrinol Metab 91:3276-3278

2. Schwartz AV, Sellmeyer DE (2007) Thiazolidinedione therapy gets complicated: is bone loss the price of improved insulin resistance? Diabetes Care 30:1670-1671

3. Rzonca SO, Suva LJ, Gaddy D, Montague DC, Lecka-Czernik B (2004) Bone is a target for the antidiabetic compound rosiglitazone. Endocrinology 145:401-406

4. Lazarenko OP, Rzonca SO, Hogue WR, Swain FL, Suva LJ, Lecka-Czernik B (2007) Rosiglitazone induces decreases in bone mass and strength that are reminiscent of aged bone. Endocrinology 148:2669-2680

5. Sottile V, Seuwen K, Kneissel M (2004) Enhanced marrow adipogenesis and bone resorption in estrogen-deprived rats treated with the PPARgamma agonist BRL49653 (rosiglitazone). Calcif Tissue Int 75:329-337

6. Sorocéanu MA, Miao D, Bai XY, Su H, Goltzman D, Karaplis AC (2004) Rosiglitazone impacts negatively on bone by promoting osteoblast/osteocyte apoptosis. J Endocrinol 183: 203-216

7. Ali AA, Weinstein RS, Stewart SA, Parfitt AM, Manolagas SC, Jilka RL (2005) Rosiglitazone causes bone loss in mice by suppressing osteoblast differentiation and bone formation. Endocrinology 146:1226-1235 
8. Schwartz AV, Sellmeyer DE, Vittinghoff E et al (2006) Thiazolidinedione use and bone loss in older diabetic adults. J Clin Endocrinol Metab 91:3349-3354

9. Grey A, Bolland M, Gamble G et al (2007) The peroxisome proliferator-activated receptor-gamma agonist rosiglitazone decreases bone formation and bone mineral density in healthy postmenopausal women: a randomized, controlled trial. J Clin Endocrinol Metab 92:1305-1310

10. Yaturu S, Bryant B, Jain SK (2007) Thiazolidinedione treatment decreases bone mineral density in type 2 diabetic men. Diabetes Care 30:1574-1576

11. Kahn SE, Haffner SM, Heise MA, ADOPT Study Group et al (2006) Glycemic durability of rosiglitazone, metformin, or glyburide monotherapy. N Engl J Med 355:2427-2443

12. Kahn SE, Zinman B, Lachin JM, Diabetes Outcome Progression Trial (ADOPT) Study Group et al (2008) Rosiglitazone-associated fractures in type 2 diabetes: an analysis from A Diabetes Outcome Progression Trial (ADOPT). Diabetes Care 31:845-851

13. Meier C, Kraenzlin ME, Bodmer M, Jick SS, Jick H, Meier CR (2008) Use of thiazolidinediones and fracture risk. Arch Intern Med 168:820-825

14. Barrett-Connor E, Siris ES, Wehren LE et al (2005) Osteoporosis and fracture risk in women of different ethnic groups. J Bone Miner Res 20:185-194
15. Bureau of National Health Insurance (2007) National Health Insurance in Taiwan. Available at www.nhi.gov.tw/english/index. asp, accessed 20 August 2009

16. Monami M, Cresci B, Colombini A et al (2008) Bone fractures and hypoglycemic treatment in type 2 diabetic patients: a casecontrol study. Diabetes Care 31:199-203

17. Loke YK, Singh S, Furberg CD (2009) Long-term use of thiazolidinediones and fractures in type 2 diabetes: a metaanalysis. CMAJ 180:32-39

18. Hampton T (2007) Diabetes drugs tied to fractures in women. JAMA 297:1645

19. GlaxoSmithKline (GSK) (2007) Clinical trial observation of an increased incidence of fractures in female patients who received long-term treatment with Avandia (rosiglitazone maleate) tablets for type 2 diabetes mellitus (Letter to Health Care Providers). Available at www.fda.gov/MedWatch/safety/2007/Avan dia_GSK_Ltr.pdf, accessed 20 March 2009

20. Takeda (2007) Observation of an increased incidence of fractures in female patients who received long-term treatment with ACTOS (pioglitazone $\mathrm{HCl}$ ) tablets for type 2 diabetes mellitus (Letter to Health Care Providers). Available at www.fda.gov/medwatch/ safety/2007/Actosmar0807.pdf, accessed 20 March 2009

21. Short R (2007) Fracture risk is a class effect of glitazones. BMJ $334: 551$ 